\title{
Cooperative and independent functions of FGF and Wnt signaling during early inner ear development
}

\author{
Kevin D. Wright, Amanda A. Mahoney Rogers, Jian Zhang and Katherine Shim * (D)
}

\begin{abstract}
Background: In multiple vertebrate organisms, including chick, Xenopus, and zebrafish, Fibroblast Growth Factor (FGF) and Wnt signaling cooperate during formation of the otic placode. However, in the mouse, although FGF signaling induces Wnt8a expression during induction of the otic placode, it is unclear whether these two signaling pathways functionally cooperate. Sprouty (Spry) genes encode intracellular antagonists of receptor tyrosine kinase signaling, including FGF signaling. We previously demonstrated that the Sprouty1 (Spry1) and Sprouty2 (Spry2) genes antagonize FGF signaling during induction of the otic placode. Here, we investigate cross talk between FGF/SPRY and Wnt signaling during otic placode induction and assess whether these two signaling pathways functionally cooperate during early inner ear development in the mouse.
\end{abstract}

Methods: Embryos were generated carrying combinations of a Spry 1 null allele, Spry2 null allele, $\beta$-catenin null allele, or a Wnt reporter transgene. Otic phenotypes were assessed by in situ hybridization, semi-quantitative reverse transcriptase PCR, immunohistochemistry, and morphometric analysis of sectioned tissue.

Results: Comparison of Spry1, Spry2, and Wnt reporter expression in pre-otic and otic placode cells indicates that FGF signaling precedes and is active in more cells than Wnt signaling. We provide in vivo evidence that FGF signaling activates the Wnt signaling pathway upstream of TCF/Lef transcriptional activation. FGF regulation of Wnt signaling is functional, since early inner ear defects in Spry 1 and Spry2 compound mutant embryos can be genetically rescued by reducing the activity of the Wnt signaling pathway. Interestingly, we find that although the entire otic placode increases in size in Spryl and Spryz compound mutant embryos, the size of the Wnt-reporter-positive domain does not increase to the same extent as the Wnt-reporter-negative domain.

Conclusions: This study provides genetic evidence that FGF and Wnt signaling cooperate during early inner ear development in the mouse. Furthermore, our data suggest that although specification of the otic placode may be globally regulated by FGF signaling, otic specification of cells in which both FGF and Wnt signaling are active may be more tightly regulated.

Keywords: Inner ear, Otic placode, Sprouty1, Sprouty2, Wnt8a, FGF, $\beta$-catenin, Cross talk

\section{Background}

The inner ears, embedded on each side of the skull, are the organs responsible for the detection of sound, linear acceleration, and rotational movement. Most of the cells that compose each inner ear and associated cochleovestibular ganglion are derived from the otic placode, a thickened region of embryonic ectoderm located lateral to each side of the developing hindbrain [1-8]. Each otic placode invaginates to form an otic cup, pinches off from the surface ectoderm to form an otic vesicle, and undergoes complex

\footnotetext{
* Correspondence: katherineshim@gmail.com

Department of Pediatrics, Children's Research Institute, Medical College of Wisconsin, Milwaukee, WI 53226, USA
}

morphogenesis to form the mature inner ear. Multiple extracellular signals, including Fibroblast Growth Factor (FGF) and Wnt, are required for formation of the otic placode $[2,4-6]$. However, the cooperative vs. distinct roles of these pathways during otic placode induction and patterning in mammals are not clear.

All cranial placodes, including the otic placode, originate from the pan-placodal region (PPR), a Ushaped, ectodermal domain located adjacent to the anterior neural plate and neural crest during gastrulation (reviewed in $[1,2,9,10])$. In multiple organisms, FGF signaling is required to specify the posterior PPR as the domain from which the otic and epibranchial placode cells will segregate, called the otic-epibranchial 
progenitor domain (OEPD, reviewed in $[2,6,8,11-13])$. In contrast, Wnt signaling is required to stabilize otic vs. epidermal/epibranchial cell fate decisions within the OEPD [11, 14-16]. In mouse, the Wnt signaling reporter, TCF/ Lef-lacZ [14, 17], is active after specification of the OEPD [14, 18, 19]. Furthermore, in chick, either activation or inhibition of Wnt signaling has no affect on formation of the OEPD $[15,18]$. In zebrafish, heterogeneous levels of pax2a or pax8 transcript and/or protein are observed in the ectoderm prior to otic placode formation, and high levels of pax2a or pax8 expression favor otic differentiation over epibranchial fates $[16,20]$. Whereas FGF signaling is required for specification of the number of Pax $2 \mathrm{a}+$ progenitor cells, the distribution of high vs. low-expressing Pax $2 \mathrm{a}+$ cells is dependent on Wnt signaling activity $[16,18]$. These data are consistent with the initiation of FGF signaling during induction of the OEPD, and the initiation of Wnt signaling later during the specification and patterning of otic placode cells within the OEPD.

Consistent with a role of Wnt signaling in otic fate decisions, otic vesicles are reduced in size when Wnt signaling is inhibited [14, 15, 21, 22]. Early ectopic activation of Wnt signaling results in the formation of enlarged or supernumerary otic vesicles due to posteriorization of the embryo leading to ectopic expression of both FGF and ectodermally-expressed otic competence factors, such as Foxi1 [21, 23, 24]. Later activation of Wnt signaling in the OEPD by expression of an activated form of the Wnt signaling effector, $\beta$-catenin, in Pax2-expressing cells in the mouse results in the expansion of otic cells at the expense of neighboring epidermal/epibranchial cells [14]. Enlarged otic vesicles are also observed in zebrafish embryos treated during somitogenesis with a chemical activator of Wnt signaling, BIO, which inhibits glycogen synthase kinase $3 \beta$ (GSK3 $\beta$ )-mediated degradation of $\beta$ catenin [16]. Similarly, enlarged or ectopic otic vesicles are observed in gain-of-function experiments in which Fgf is overexpressed in multiple organisms (reviewed in [13], see also [25]). However, misexpression of Fgf can also lead to an opposite phenotype - the inhibition of otic differentiation $[15,25,26]$. Combined, these data indicate that the timing, dosage and spatial distribution of both FGF and Wnt signaling during the induction of the otic placode must be tightly regulated $[15,16,21,25,26]$.

In multiple organisms, FGF and Wnt signaling cooperate during induction of the otic placode. In chick, Wnt8c functions synergistically with FGF signaling to induce otic fate [27]. In Xenopus, whereas inhibition of either FGF or Wnt signaling alone reduces the ability of neural plate tissue to induce the expression of the otic marker, pax8, in explant cultures, simultaneous inhibition of both FGF and Wnt signaling almost completely abrogates pax8 induction [28]. Furthermore, morpholino (MO) knockdown of combinations of Wnt and Fgf genes (eg. Fgf8-MO + Wnt8-MO) in
Xenopus results in greater reduction of otic expression of pax8 and sox 9 than single morpholino knock-down of Fgf or Wnt alone [28]. Similarly, in zebrafish, morpholino knock-down of wnt8b in $f g f 3$ mutant embryos produces otic vesicles that are smaller than otic vesicles produced after inactivation of either wnt8b or $f g f 3$ alone [22].

In mice, expression of Wnt8a in the hindbrain is reduced in $\mathrm{Fgf3}^{-/-}$; $\mathrm{FgflO}^{-/-}$double mutant embryos and otic vesicles are reduced or absent [29]. Sprouty genes encode antagonists of receptor tyrosine kinase (RTK) signaling, including FGF signaling. We have previously demonstrated that in Spry $1^{-/-}$; Spry $2^{-/-}$double mutant embryos, the otic placode is larger and Wnt8a expression is expanded [18]. Combined, these data demonstrate that FGF signaling regulates the Wnt pathway at the level of Wnt8a expression. However, recent data demonstrate that otic placodes form normally in $W n t 8 a^{-/-}$knockout embryos [30]. Furthermore, Vendrell et al. demonstrate that the otic placode and vesicle form normally upon combinatorial inactivation of Wnt8a and either Fgf3 or

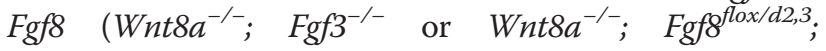
Mesp1Cre/+ embryos). Thus, there is currently little evidence in mammals that cross talk between FGF and Wnt signaling is functionally required during otic placode induction.

Inactivation of Spry genes results in over-activity of receptor tyrosine kinase signaling in its normal tissue context. Here, we took advantage of the enlarged placode in Spry $1^{-/-}$; Spry $2^{-/-}$mutants to assess expression of Wnt pathway components and Wnt signaling activity in tissues in which FGF-response is elevated. This allowed us to further characterize the relationship between FGF and Wnt signaling during otic placode induction and to assess whether these two signaling pathways functionally cooperate during early mammalian inner ear development.

\section{Methods \\ Mouse lines}

Mouse lines carrying null or floxed alleles of Spry1 [31], Spry2 [32], the conditional $\beta$-catenin ${ }^{\text {flox/flox }}$ allele ([33], purchased from the Jackson Laboratory, B6.129-Ctnnb1 $1^{\text {tm2Kem }}$, KnwJ, stock number 004152), $\beta$-actin-Cre [34] and TCF/ Lef-lacZ reporter mice [17] were maintained and genotyped as described. Spry mutant embryos were generated by crossing $\beta$-actin cre/ $\beta$-actin cre; Spry $1^{-/+}$; Spry $2^{-/+}$males to Spry $1^{\text {floxfllox}} ;$ Spry $2^{\text {flox/flox }}$ females. For all experiments in which Spry1 ${ }^{-/+}$; Spry $2^{-/+}$mutant embryos were used as littermate control embryos, comparison was made to CD-1 embryos to verify the absence of defects. Wnt reporter activity was assessed in Spry-deficient embryos by crossing $\beta$-actin cre/ $\beta$-actin cre; Spry1 $1^{-/+} ;$Spry $2^{-/+}$ males to Spry flox/flox $_{\text {Spry }} 2^{\text {flox/flox }}$; TCF/Lef-lacZ/+ or Spry $1^{\text {flox/flox }} ;$ Spry $2^{\text {flox/flox }} ;$ TCF/Lef-lacZ/TCF/Lef-lacZ females. $\beta$-catenin ${ }^{-/+}$animals were generated by 
crossing $\beta$-catenin ${ }^{\text {flox/flox }}$ mice to $\beta$-actin-Cre/ $\beta$-actinCre animals. Resulting progeny were bred to both remove the $\beta$-actin-Cre allele and to generate Spry $1^{\text {flox }}$ ${ }^{\text {flox }}$; Spry $2^{\text {flox flox }} ; \beta$-catenin ${ }^{-/+}$animals. Genetic interaction experiments were performed by generating embryos from a $\beta$-actin cre/ $\beta$-actin cre; Spry $1^{-/+}$; Spry2 $2^{-/+}$

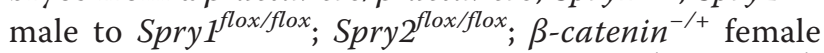
cross. CD-1 embryos were used as Spry $^{+/+} ;$Spry2 $2^{+/+}$ controls to assess the size of the Wnt8a expression domain. All procedures were approved by the Institutional Animal Care and Use Committee at the Medical College of Wisconsin.

\section{In situ hybridization}

Embryos were staged so that noon on the day of vaginal plug detection was designated as embryonic day (E) 0.5. Embryos were dissected in phosphate buffered saline, $0.1 \%$ Tween-20 and fixed by immersion in $4 \%$ paraformaldehyde at $4{ }^{\circ} \mathrm{C}$ for one hour. Whole-mount in situ hybridization was performed according to standard protocols, using the following digoxigenin-labeled probes: Spry1, Spry2, Pax8, Foxi2, Wnt8a, Wnt1, Wnt3a, Wnt6, $F z d 1$, and Fzd8. All probes were tested on CD-1 wildtype embryos, and expression patterns were compared to published images. Scoring of changes in gene expression patterns or levels was based upon comparison of all embryos of a particular genotype with all embryos of another genotype. Photography of whole-mount embryos was done at the same exposure, using a Zeiss Discovery V.12 microscope.

\section{Microdissection and semi-quantitative reverse transcriptase PCR}

OEPD, underlying mesenchyme, and adjacent neural ectoderm were microdissected from 5-7 s embryos using tungsten needles and fine forceps [29]. Tissues from each embryo were stored separately in TRIzol reagent (Life Technologies) at $-80{ }^{\circ} \mathrm{C}$ before genotyping. Tissues from 2 embryos of the same genotype were pooled. RNA was isolated and reverse-transcribed using the SuperscriptIII first-strand synthesis kit and oligo-dT primers (Life Technologies). For each gene, primers were validated for specificity by inclusion of "no reverse transcriptase" samples and ability to amplify a single band of the correct size. For each primer pair, test PCRs were performed across a series of cycle numbers to identify the linear range prior to plateau [35]. Experimental PCRs were performed at a cycle number in the linear range using GoTaq 2X polymerase (Promega). All PCR reactions were performed at least twice on 2-3 biological replicates (4-6 embryos). Gapdh was used as a pipetting control.

Primer sequences were: Gapdh forward 5'- GGGCT GGCATTGCTCTCAATGACAACTTT-3' and reverse

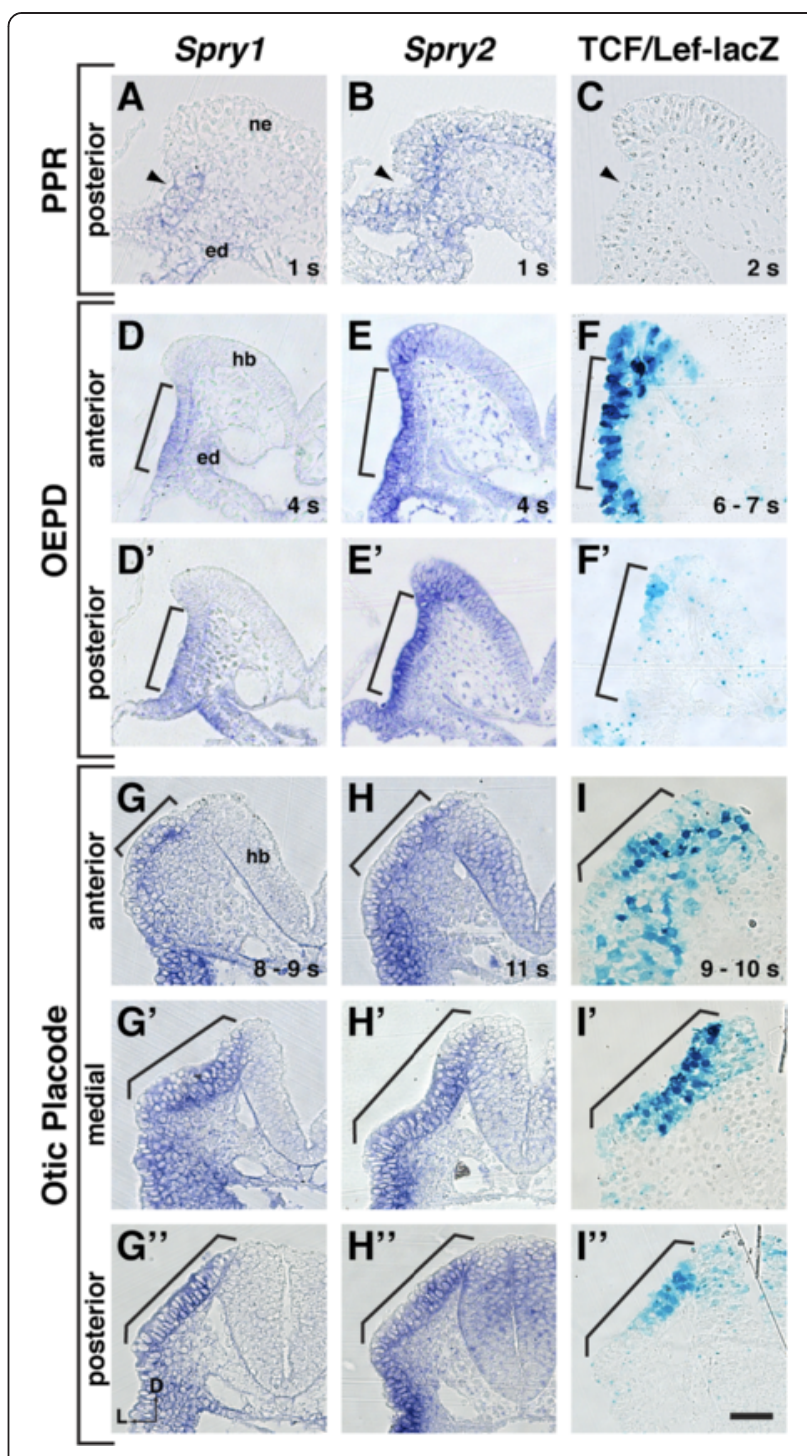

Fig. 1 Comparison of Spry1, Spry2, and Wnt reporter expression domains from PPR to otic placode stages. In situ hybridization analysis of Spryl and Spry2 expression domains compared to Wnt reporter activity in TCF/Lef-lacZ embryos at the stages indicated. Transverse sections are shown, dorsal oriented to the top. a - c Spry 1 expression, Spry2 expression, and TCF/Lef-lacZ reporter activity at early somite stages in the posterior PPR. Arrowhead indicates the presumptive otic/ epibranchial region. Little or no TCF/Lef-lacZ reporter activity is detected in the posterior PPR at this stage $(\mathbf{c}) .\left(d-f^{\prime}\right)$ Spry 1 expression, Spryz expression, and TCF/Lef-lacZ reporter activity in anterior $(\mathbf{d}-\mathbf{f})$ and posterior $\left(d^{\prime}-f^{\prime}\right)$ transverse sections through the OEPD. The entire OEPD is bracketed. ( $\left(\mathrm{-}-\mathrm{i}^{\prime \prime}\right)$ Spry1 expression, Spry2 expression, and TCF/ Lef-lacZ reporter activity in anterior $(\mathbf{g}-\mathbf{i})$, medial $\left(g^{\prime}-i^{\prime}\right)$, and posterior $\left(g^{\prime \prime}-i^{\prime \prime}\right)$ transverse sections through the otic placode (bracketed). Abbreviations: neural ectoderm (ne), endoderm (ed), hindbrain (hb). Scale bar, $50 \mu \mathrm{m}$

5' - CACCCTGTTGCTGTAGCCGTATTCAT-3'; Wnt1 forward 5'-CAGCTGGGTTTCTACTACGTT' 3 ' and reverse 5'-CAGACTCTTGGAATCCGTCAA-3'; Wnt3a forward $5^{\prime}$-TGCCATGAACCGTCACAA- 3 ' and reverse 
5'-CAGCAGGTCTTCACTTCACA-3'; Wnt6 forward $5^{\prime}$ - GCCAGACTGCGGTAGAG-3' and reverse 5'- GT AGGATCCATGACCAAGGG-3'; Wnt8a forward $5^{\prime}$-GG TGGAATTGTCCTGAGCAT-3' and reverse $5^{\prime}$-GTTC TTGGTGACTGCGTACA; Wnt3 forward 5'-GCCAAG AGTGTATTCGCATCTA-3' and reverse 5' -TCATGGG ACTTCGATGAATGG-3'; Wnt5a forward 5'-TGGC AGGGTGATGCAAATA-3' and reverse 5' -CTGCAGC CACAGGTAGAC-3'; Wnt5b forward 5' - CGAGAGCG TGAGAAGAACTTT-3' and reverse $5^{\prime}$ - GGCGACATC AGCCATCTTAT-3'; Wnt7b forward 5'- GGATGCCC GTGAGATCAAA-3' and reverse 5' - GACACACCGTG ACACTTACA-3'; Fzd3 forward 5' - GCTTTGAATGG GCCAGTTT-3' and reverse 5' - TCAGGAGTGACT GAGCAAAG-3'; $F z d 7$ forward 5' - AAAGGCAGTG GCCGAAA-3' and reverse 5' $^{\prime}$ - TCTCTCTCTGCTGGT CTCAA-3'.

\section{LacZ stain}

To detect $\beta$-galactosidase activity in embryos carrying the TCF/Lef-lacZ transgene, embryos were dissected in phosphate buffered saline, $0.1 \%$ Tween-20, then fixed and washed. Embryos were incubated with X-gal as described in [17] except that incubations were performed at room temperature for varying lengths of time. All OEPD-stage embryos and otic placode-stage embryos that were sectioned for measurement analysis (Fig. 4e, f) were incubated in X-gal overnight. To assess differences in intensity of staining at otic placode stages, embryos were incubated in X-gal for 60 mins. (Additional file 2: Figure S2) or 90 mins. (Additional file 1: Figure S1). Whole-mount embryos were photographed using a Zeiss Discovery V.12 microscope.

To compare Spry1, Spry2, and TCF/Lef-lacZ reporter expression domains, embryos were stained in wholemount, post-fixed overnight in $4 \%$ paraformaldehyde, then embedded in JB-4 plastic (Polysciences). Serial transverse sections were cut at $6 \mu \mathrm{m}$ thickness. To compare staining patterns between embryos stained for different markers, sections were aligned by morphological criteria. The posterior PPR was identified as the ectoderm present in sections in which the notochord was visible and the intra-embryonic coelomic cavity had split into two left and right horns. At the OEPD stage, the anterior end was designated as the start of the appearance of cubiodal vs. squamous epithelium and the posterior end of the OEPD was designated at the transition from cubiodal to squamous epithelium. Sections containing the OEPD coincided with the presence of the first branchial pouch and OEPD was absent in sections where the entrance to the foregut diverticulum was visible. The otic placode was defined based upon the presence of a thickened pseudostratified epithelium, at least two nuclei thick.

\section{E-cadherin stain}

For E-cadherin immunostaining, embryos were dissected at E9.0 to E9.5 and were stained in whole mount with anti-E-Cadherin antibody (Life Technologies, 1:1000 dilution). A biotinylated anti-rat IgG secondary antibody (Vector Laboratories), followed by Vectastain Elite ABC (Vector Laboratories) and TSA fluorescein tyramide reagent (PerkinElmer) amplification, were used to detect anti-E-cadherin as described [36]. Embryos were photographed using a Zeiss Observer Z1 inverted microscope.

To compare E-cadherin staining intensity, mutant and control embryos were fixed in $4 \%$ paraformaldehyde at $4{ }^{\circ} \mathrm{C}$ overnight, cut by vibratome, then stained with anti-Ecadherin antibody (Life Technologies, 1:1000 dilution), using an Alexa-488-conjugated anti-rat IgG secondary antibody (Life Technologies, 1:1000 dilution) as described [37]. Images were collected using a Zeiss LSM510 laser scanning confocal microscope (data not shown).

\section{Morphometric analysis}

To measure the size of the otic placode, reporter-positive, and reporter-negative domains, embryos were stained for reporter activity in whole-mount, then embedded in JB-4 plastic (Polysciences). Serial transverse, $6 \mu \mathrm{m}$-thick sections were cut. Embryos in which more than 4 sections were lost during microtomy were excluded. All sections containing the otic placode were photographed, and images were used to measure the medial-to-lateral length of the otic placode using Image J software. The otic

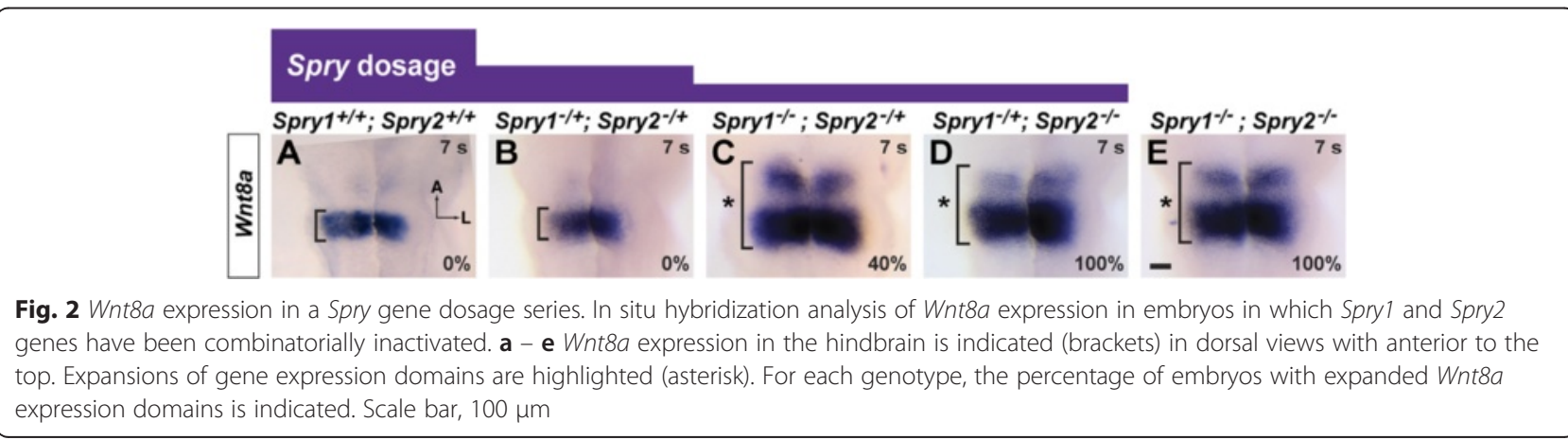



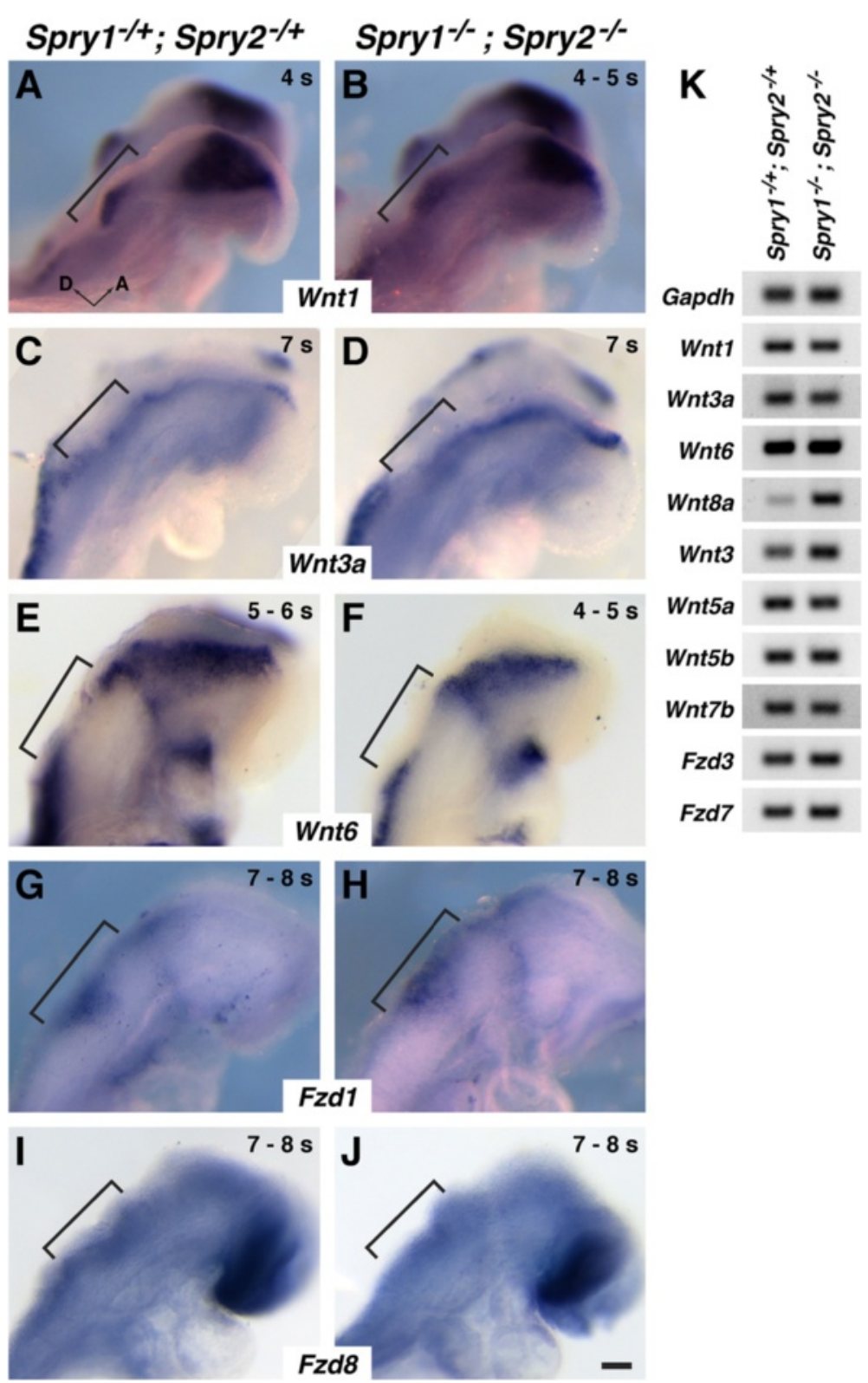

Fig. 3 Expression patterns of genes encoding Wnt ligands and receptors are unchanged in Spry $1^{-1-}$; Spry $2^{-1-}$ embryos. a - j In situ hybridization analyses of genes encoding Wnt ligands and receptors in Spry ${ }^{-1-}$; Spry $2^{-/-}$embryos and controls at OEPD or early otic placode stages. Lateral views of wholemount embryos are shown; embryos are oriented as indicated. Pre-otic regions are bracketed. $\mathbf{k}$ Semi-quantitative reverse-transcriptase PCR analyses of candidate Wht and Fzd transcript levels in RNA collected from $5-7 \mathrm{~s}$ hindbrain and OEPD-containing tissue microdissected from the genotypes indicated. Wnt genes (Wnt1, Wnt3a, Wnt6, and Wnt8a) with known expression adjacent to the OEPD are controls. Scale bar, $100 \mu \mathrm{m}$

placode was identified based on its pseudostratified epithelial morphology, and was measured if it was at least two nuclei thick. Using Image J, a freehand line was drawn along the basal surface of the otic epithelium, following the curvature of the otic placode. For the majority of embryos, both the left and right side of the embryos were measured.
For the total placode, reporter-positive, and reporternegative domains, average cross-sectional basal surface areas $\left(\mathrm{mm}^{2}\right)$ were calculated as the sum of the medial-tolateral lengths from all sections multiplied by $0.006 \mathrm{~mm}$ (the thickness of each section). Fold area changes were calculated by normalizing the area for each placode by the mean area in Spry $1^{-/+}$; Spry $2^{-/+}$controls. Significance of 


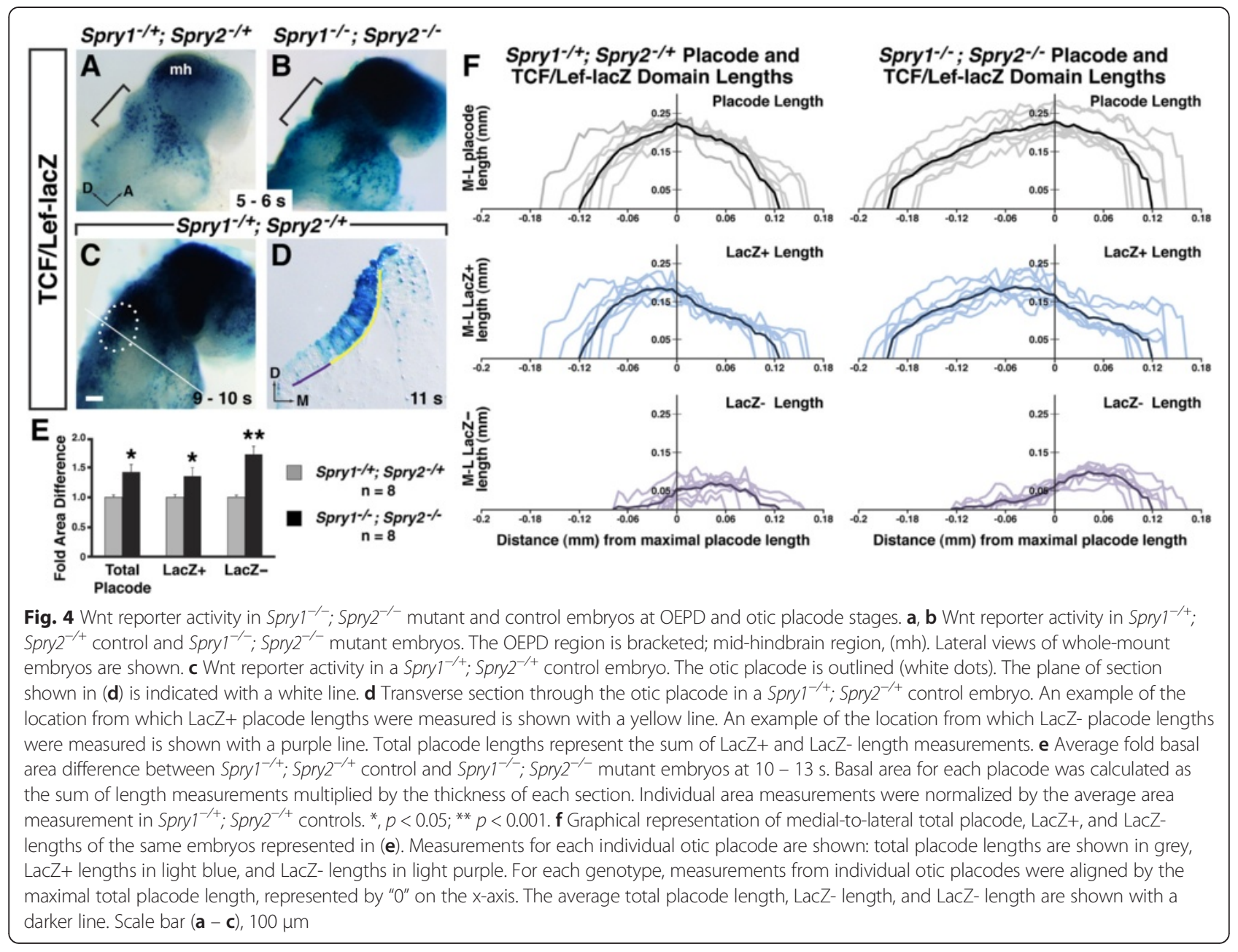

difference between genotypes was measured by one-way analysis of variance (ANOVA).

Medial-to-lateral, total placode measurements were graphed by aligning from the widest length, which was centered on " 0 " at the y-intercept. Measurements from each individual placode were graphed, along with average measurements. Start and end of the averaged plot were determined by calculating the average anterior and posterior distances from the $y$-intercept. Medial-to-lateral reporter-positive and reporter-negative measurements were graphed to align with the total placode measurements. Therefore, all graphs were aligned by the maximal width of the total placode.

Anterior-posterior lengths of $\operatorname{Spry} 1^{-/+} ; \operatorname{Spry} 2^{-/+} ; \beta-$ catenin $^{+/+}$embryos, Spry1 $1^{-/-}$; Spry $2^{-/-} ; \beta$-catenin ${ }^{+/+}$embryos, and Spry1 ${ }^{-/-}$; Spry $2^{-/-}$; $\beta$-catenin ${ }^{-/+}$embryos were measured by identifying the number of transverse sections containing an otic placode and multiplying by the thickness of each section $(0.01 \mathrm{~mm})$. Otic placodes were identified as thickened epithelium, at least 2 cells thick, that did not express Foxi2.

\section{Results}

Spry 1 and Spry 2 were expressed earlier and in a broader domain than a Wnt reporter

We previously demonstrated that Spry1 and Spry2 were expressed in the OEPD ectoderm and underlying mesenchyme, and that expression of both genes restricted to the ectoderm when the otic placode was morphologically distinct [18]. Groves and colleagues have demonstrated that the Wnt signaling reporter, TCF/Lef-lacZ [17], is active after induction of the OEPD, marked by Pax 2 expression $[14,19]$. Furthermore, the TCF/Lef-lacZ reporter is expressed in anterior and medial regions of the otic placode, but is absent from a posterior-ventrolateral domain [14]. To define the spatial and temporal relationship between Spry1 and Spry2 expression and Wnt signaling activity, we re-examined Spry 1 and Spry2 expression by in situ hybridization to 1) determine the timing of onset of expression of these genes in the PPR or OEPD and 2) determine whether these genes were expressed in posterior regions of the OEPD and otic placode, where TCF/LeflacZ reporter activity is absent. 
We found that Spry1 and Spry 2 transcripts were present broadly in the PPR, beginning at pre-somite stages. In the posterior PPR, both genes were expressed strongly in the ectoderm, with additional expression in the underlying mesenchyme and endoderm (Fig. 1a, b). Spry 2 transcript was also detected in neural ectoderm (Fig. 1b). Consistent with previous reports [14, 19], TCF/Lef-lacZ reporter activity was not detected at this stage in the PPR (Fig. 1c). By $3-5 \mathrm{~s}$, TCF/Lef-lacZ reporter activity is first detected in the OEPD $[14,19]$. At this stage, Spry1 and Spry 2 transcripts were detected broadly throughout the OEPD in ectoderm, underlying mesenchyme, and endoderm (Fig 1d, d', e, e'). Whereas expression of Spry1 and Spry2 coincided with TCF/Lef-lacZ reporter activity in the anterior ectoderm of the OEPD (Fig. 1d - f), Spry1 and Spry2 expression extended further into posterior and ventrolateral regions compared to TCF/Lef-lacZ reporter activity (Fig. 1d' - f'). In this posterior region, TCF/Lef-lacZ activity was restricted to a few cells adjacent to the developing hindbrain (Fig. 1f'). TCF/Lef-lacZ activity was also detected in migrating neural crest cells, which did not strongly express Spry1 or Spry2 (data not shown). When the otic placode became morphologically distinct, Spry1 and Spry2 transcripts were detected throughout the otic placode (Fig. 1g - g", h - h", brackets). Whereas expression of Spry1 and Spry2 was not detected in surface ectoderm anterior or posterior to the placode, expression extended ventrally from the otic placode in epidermal and epibranchial progenitor cells (Fig. $1 \mathrm{~g}-\mathrm{g}$ ", $\mathrm{h}-\mathrm{h}$ "). Consistent with previous findings [14], Wnt reporter activity was detected in anterior and medial regions of the otic placode, but was absent from a posterior-ventrolateral region (Fig. 1i - i"). In summary, Spry1 and Spry2 were expressed in the PPR, prior to the onset of Wnt reporter activity. At the time of onset of Wnt reporter activity in the OEPD and continuing through otic placode stages, Spry1 and Spry2 expression domains overlapped with Wnt reporter expression in anterior-medial regions but extended beyond this domain posteriorly and ventrolaterally.

\section{Expansion of hindbrain Wnt8a expression correlated with otic placode expansion}

We previously demonstrated that the Wnt8a expression domain [14] is expanded in the hindbrain adjacent to the OEPD in Spry1 and Spry2 compound mutant embryos (Spry1 $1^{-/-}$; Spry2 $2^{-/-}$or "Spry-deficient" embryos) at $6-7 \mathrm{~s}$, indicating that Spry genes regulate Wnt expression [18]. Conversely, hindbrain Wnt8a expression is absent in embryos in which Fgf3 and Fgf10 have been combinatorially inactivated $\left(\mathrm{Fgf3}^{-/}\right.$; Fgf10 -/- mutant embryos and a subset of $\mathrm{Fgf3}^{-/-} ; \mathrm{FgflO}^{-/+}$ mutant embryos), as well as in a subset of $\mathrm{Fgf3}^{-/-}$single mutant embryos [29]. Since the otic placode forms normally in $F g f 3^{-/-}$single mutants [29, 38-41], the absence of Wnt8a expression in a subset of these mutant embryos is consistent with the finding that Wnt8a alone is not required for formation of the otic placode [30].

We have shown that generation of a Spry gene dosage series by combinatorial inactivation of Spry1 and Spry2 results in enlargement of the otic placode in a subset of Spry $1^{-/-}$; Spry2 $2^{-/+}$and Spry1 $1^{-/+} ;$Spry2 $2^{-/-}$mutant embryos [42]. The penetrance of otic placode enlargement increased with increasing loss of Spry gene function, such that $33-50 \%$ of Spry $1^{-/-}$; Spry $2^{-/+}$mutant embryos, $71-89 \%$ of Spry1 $1^{-/+}$; Spry2 $2^{-/-}$mutant embryos, and $83-100 \%$ of Spry-deficient $\left(\right.$ Spry $1^{-/}$; Spry $2^{-/-}$) embryos had otic placode phenotypes [42]. To determine whether, as in $\mathrm{Fgf3}^{-/-}$mutant embryos, otic placode phenotypes are de-coupled from the size of the Wnt8a expression domain, we examined Wnt8a expression in the Spry gene dosage series. We performed in situ hybridization on whole mount embryos to detect Wnt8a transcript at $6-8 \mathrm{~s}$, prior to the stage when expansion of the otic placode was observed [18, 42]. Consistent with our previous findings, in $100 \%$ of Spry-deficient embryos, Wnt8a expression in the hindbrain was expanded compared to wild-type controls (compare Fig. 2a to e, 6 out of 6 Spry $1^{-/-}$; Spry $2^{-/-}$double mutant embryos, $n=10$ Spry $1^{+/+}$; Spry $2^{+/+}$controls). In $100 \%$ of Spry $1^{-/+}$; Spry $2^{-/-}$embryos (Fig. 2d, 5 out of 5 embryos) and $40 \%$ of Spry $1^{-/-}$; Spry2 $2^{-/+}$embryos (Fig. 2c, 2 out of 5 embryos) Wnt8a expression in the hindbrain was expanded. No expansion of Wnt8a expression was observed in Spry1 $1^{-/+}$; Spry2 $2^{-/+}$compared to wild-type embryos (compare Fig. 2a and b, 0 out of 4 embryos). Thus, in a Spry gene dosage series, the frequency at which expanded Wnt8a expression was observed was consistent with the frequency at which otic placode expansion was observed. To confirm that enlargement of the otic placode correlated with enlargement of Wnt8a expression, we performed in situ hybridization on $6-8 \mathrm{~s}$ whole mount embryos using a mixture of a Wnt8a and Pax 8 probes to visualize the Wnt8a expression domain and the otic placode in the same embryo. For each genotype in the Spry gene dosage series, if the otic placode appeared larger, the Wnt8a expression in the hindbrain also appeared expanded. Conversely, if the otic placode appeared a normal size, the Wnt8a expression domain also appeared normal (data not shown, $n=3$ embryos for each of the following genotypes: Spry $1^{-/+}$; Spry $2^{-/+}$control embryos, Spry1 $1^{-/}$; Spry2 $2^{-/+}$embryos, Spry1 $1^{-/+}$; Spry2 $2^{-/-}$ embryos, and Spry $1^{-/-}$; Spry $2^{-/-}$double mutant embryos).

Therefore, in Spry mutant embryos, unlike Fgf mutants, the size of the otic placode correlated with the size of the $W n t 8 a$ expression domain. Furthermore, Fgf genes have differential roles in $W n t 8 a$ regulation, with $F g f 3$ playing a 
more significant role than Fgf10 [29]. In contrast, Spry1 and Spry 2 regulation of both Wnt8a expression and otic placode size may be more functionally redundant.

\section{Inactivation of Spry1 and Spry2 did not affect expression of other genes encoding Wnt ligands or Fzd receptors} To determine whether Spry genes regulate the expression of other Wnt genes besides Wnt8a or genes that encode Wnt receptors, we determined the expression of known hindbrain-expressed Wnt genes and OEPD-expressed Frizzled $(F z d)$ genes in Spry-deficient and control embryos. Prior to otic placode formation, Wnt1 (Fig. 3a), Wnt3a (Fig. 3c), and Wnt6 (Fig. 3e) are expressed adjacent to the OEPD in dorsal neural ectoderm, in the region of premigratory neural crest cells $[19,29]$. We found no gross difference in expression patterns of these genes in Sprydeficient embryos compared to control embryos, and minor differences in expression domain were not consistent between genotypes (compare Fig. 3a, c, e to Fig. 3b, d, f; Wnt1, $n=6$ Spry $1^{-/}$; Spry $2^{-/-}$mutant embryos; Wnt3a, $n=9$ Spry1 $1^{-/} ;$Spry2 $2^{-/-}$mutant embryos; Wnt6, $n=4$ Spry $1^{-/-}$; Spry2 $2^{-/-}$mutant embryos). In addition, genes encoding two Wnt receptors, $F z d 1$ and $F z d 8$, are expressed in pre-otic tissue at $6-8 \mathrm{~s}$ ([29], Fig. 3g, h). No gross difference in the expression of $F z d 1$ or $F z d 8$ was found in Spry-deficient embryos compared to controls (compare Fig. 3g, i to Fig. 3h, j; Fzd1, $n=6$ Spry $1^{-/-}$; Spry2 $2^{-/-}$mutant embryos, Fzd8, $n=7$ Spry1 $1^{-/}$; Spry2 $2^{-/-}$mutant embryos).

Furthermore, we microdissected tissue containing the OEPD and adjacent neural ectoderm from 5 - 7 s Sprydeficient and control embryos. We assessed expression levels of additional candidate Wnt and Fzd genes expressed in neural ectoderm using semi-quantitative reverse transcriptase PCR (RT-PCR). As confirmation of the microdissection technique and consistent with in situ hybridization analyses, RT-PCR indicated that Wnt1, Wnt $3 a$ and Wnt6 expression levels were unaffected in Spry-deficient embryos (Fig. 3k). Furthermore, consistent with in situ hybridization results, RT-PCR suggested that expression of Wnt8a was elevated in Spry-deficient embryos (Fig. 3k). We examined additional candidate Wnt and $F z d$ genes that have neural ectoderm or otic expression at E8.5 or E9.5: Wnt3 [1], Wnt5a [1, 3, 5], Wnt5b [5], $W n t 7 b$ [1], Fzd3 [11], and $F z d 7$ [11]. Expression levels of all of these genes were comparable in Spry-deficient and control embryos (Fig. 3k). Thus, of the genes that encode

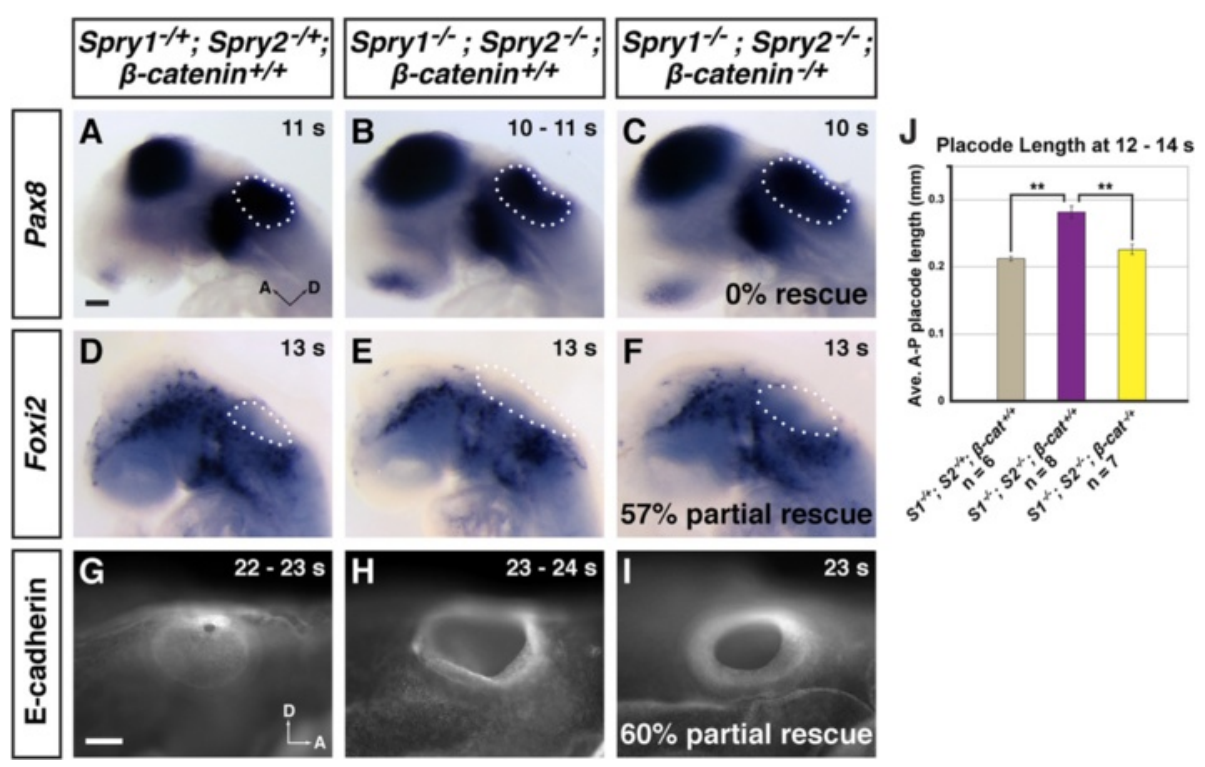

Fig. 5 Partial rescue of otic phenotypes Spry ${ }^{-1-}$; Spry $2^{-/-}$mutants by reducing the dosage of $\beta$-catenin. $\mathbf{a}-\mathbf{c}$ In situ hybridization analysis to detect Pax8 expression in the otic placode (outlined with white dots). c No rescue of otic placode expansions were observed in Spry $1^{-1-}$; Spry $2^{-1-}$; $\beta$-catenin ${ }^{-/+}$embryos, as indicated. $\mathbf{d}$ - $\mathbf{f}$ In situ hybridization analysis to detect Foxi2 expression in epidermal/epibranchial cells surrounding the

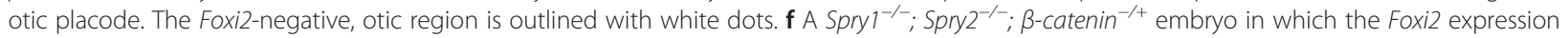
pattern appeared more similar to normal control embryos (d), rather than Spry-deficient embryos (e). The percentage of $\operatorname{Spry} 7^{-1-} ; \operatorname{Spry2^{-1}}$; $\beta$-catenin ${ }^{-1+}$ embryos with partial rescue of the Foxi2 expression pattern is indicated. $\mathbf{g}-\mathbf{i}$ E-cadherin antibody stain on whole-mount embryos to reveal the extent of closure of the otic cup. i A Spry $1^{-1-} ;$ Spry $^{-1-} ; \beta$-catenin ${ }^{-1+}$ embryo in which the otic cup is more closed than any Spry-deficient control (see H). The percentage of Spry $7^{-1-}$; Spry $2^{-1-} ; \beta$-catenin ${ }^{-/+}$embryos in which otic cup closure was partially rescued is indicated. $\mathbf{j}$ Average

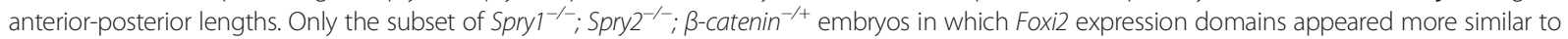
normal were selected for length measurement. Scale bar $(\mathbf{a}-\mathbf{f}),(\mathbf{g}-\mathbf{i}), 100 \mu \mathrm{m}$ 
Wnt ligands and receptors known to be expressed in or adjacent to the OEPD, only Wnt8a expression is Spryregulated.

\section{Wnt signaling activity was increased in Spry $1^{-/} ;$Spry $2^{-/-}$ mutants}

To determine whether cellular response to Wnt signaling was affected in Spry-deficient embryos, we bred the TCF/ Lef-lacZ transgene into the Spry mutant background, and assayed for lacZ reporter activity in $\operatorname{Spry1^{-1}}{ }^{\text {; }} \operatorname{Spry} 2^{-1-}$; TCF/Lef-lacZ/+ mutant embryos compared to Spry1 ${ }^{-/+}$; Spry $^{-/+}$TCF/Lef-lacZ/+ controls. In control embryos, at the onset of TCF/Lef-lacZ reporter activity in the OEPD at $3-6 \mathrm{~s}$, reporter-positive cells were detected in an anterior-dorsal domain (Fig. 4a, $n=7$ ). In Spry1 ${ }^{-/-}$; Spry2 $2^{-/}$; TCF/Lef-lacZ/+ mutant embryos TCF/Leflac $Z$ reporter activity initiated in the OEPD at the same time as controls, but was qualitatively elevated (Fig. 4b, $n=7)$. In addition, more cells in the OEPD were reporter-positive, such that individual reporter-positive cells could not be easily distinguished from neighboring reporter-positive cells. The increase in TCF/Lef-lacZ reporter activity in the Spry-deficient background was not specific to the OEPD: all TCF/Lef-lacZ-positive domains in the whole mount embryo, including the mid-hindbrain (Fig. 4a, b) and posterior embryonic regions (not shown)

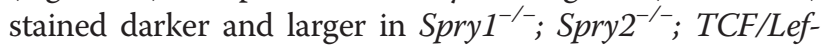
lacZ/+ mutant embryos. However, the gross pattern of the TCF/Lef-lacZ-positive domain was similar in Spry-deficient embryos compared to controls. At the otic placode stage $(9-13 \mathrm{~s})$, TCF/Lef-lacZ staining was also darker and broader in Spry1 ${ }^{-/-}$; Spry2 $2^{-/-}$; TCF/Lef-lacZ/+ mutant embryos compared to controls (see Additional file 1: Figure S1C, D; $n=3$ mutant embryos, $n=2$ controls). These data indicate that Wnt signaling activity is elevated in Spry-deficient embryos in both the OEPD and otic placode.

\section{Effect of loss of Spry function on size of the Wnt-reporter- positive and Wnt-reporter-negative domains}

In the otic placode, TCF/Lef-lacZ reporter activity is detected in an anterior-dorsal domain but is absent from a posterior-ventrolateral region ([14], Fig. 4c). In Spry-deficient embryos, the otic placode is increased in size ([18]). To determine whether the size of the TCF/Lef-lacZ reporter domain increased in proportion with the entire otic placode in Spry-deficient embryos, we performed a morphometric analysis. We cut transverse sections of $9-13 \mathrm{~s}$ Spry $1^{-/-}$; Spry2 $2^{-/-}$; TCF/Lef-lacZ/+ mutant embryos and Spry $1^{-/+}$; Spry $2^{-/+}$TCF/Lef-lacZ/+ controls that had been stained for LacZ activity. After photography of each section through the otic placode, the medial-lateral lengths of the total placode, LacZ-positive (Fig. 4d, yellow line), and LacZ-negative (Fig. 4d, purple line) regions were measured by tracing along the basal surface of the otic placode using ImageJ. Basal surface area for each region (LacZ-positive, LacZ-negative, or total placode) was calculated by summing all basal length measurements obtained from each section and multiplying this sum by the thickness of the sections $(6 \mu \mathrm{m})$.

In Spry-deficient embryos, the mean basal surface area of the total otic placode was 1.42-fold larger than the area in control embryos (Fig. 4e, $0.055 \pm 0.013 \mathrm{~mm}^{2}$ in Spry-deficient embryos compared to $0.039 \pm 0.005 \mathrm{~mm}^{2}$ in control embryos, $p=0.006, n=8$ placodes for each genotype). For the LacZ-positive, Wnt-responsive domain, the mean basal surface area in Spry-deficient embryos was 1.36-fold larger than the LacZ-positive area in controls (Fig. 4e, $0.043 \pm 0.012 \mathrm{~mm}^{2}$ in Spry-deficient embryos compared to $0.032 \pm 0.004 \mathrm{~mm}^{2}$ in control embryos, $p=0.022$ ). In contrast, the mean basal surface area of the LacZ-negative domain in Spry-deficient embryos was 1.72-fold larger than the LacZ-negative domain in control embryos (Fig. 4e, $0.012 \pm 0.003 \mathrm{~mm}^{2}$ in Spry-deficient embryos compared to $0.007 \pm 0.001 \mathrm{~mm}^{2}$ in control embryos, $p=$ 0.0001). Thus, in Spry-deficient embryos, the region of the otic placode with no Wnt reporter activity increased in size to a greater extent than the Wnt-responsive region. As a result, the LacZ-negative domain occupied $18 \%$ of the otic placode in control embryos and $22 \%$ of the otic placode in Spry-deficient embryos.

To graphically represent the size and shape of the otic placode, we plotted medial-lateral length measurements from anterior to posterior. We aligned measurements from different individual placodes of the same genotype by the maximal medial-lateral length, a measurement that can be unequivocally identified (Fig. 4f, top graphs, maximal medial-lateral length denoted as " 0 " and designated as the $y$-intercept). Graphical representation revealed a shape change in Spry-deficient otic placodes. We found that the increased size of the otic placode in Spry-deficient embryos (see Fig. 4e) was primarily due to an anterior-posterior expansion, rather than medial-lateral (Fig. 4f, top graphs). The average anterior-posterior length of the otic placode in Spry-deficient embryos was $0.319 \pm 0.034 \mathrm{~mm}$ compared to $0.240 \pm 0.027 \mathrm{~mm}$ in control embryos $(p=0.0002, n=8$ placodes for each genotype). Furthermore, in control embryos, the anterior and posterior regions of the otic placode were distributed symmetrically on either side of the widest point (Fig. 4e, top left graph). In contrast, in Spry-deficient embryos, the otic placode was asymmetrically shaped around the widest point, with anterior regions elongated.

Measurements of the LacZ-positive and LacZ-negative domains were graphed to align with total placode plots. Thus, for each placode, plots of total placode, LacZpositive and LacZ-negative measurements were all aligned from the same point of maximal medial-lateral total placode width. In Spry-deficient embryos, the shape 
of the Wnt-responsive, LacZ-positive domain followed the shape of the otic placode, and was more elongated anteriorly (Fig. 4f, middle graphs). Consistent with a disproportionate enlargement of the LacZ-negative domain in Spry-deficient embryos, the LacZ-negative domain appeared larger and extended further anteriorly than the LacZ-negative domain in control embryos (Fig. 4f, bottom graphs). The anterior-posterior length of the LacZnegative domain was $0.200 \pm 0.033 \mathrm{~mm}$ in Spry-deficient embryos compared to $0.135 \pm 0.028 \mathrm{~mm}$ in controls, $p=$ 0.0009). Together, these data suggest that in Spry-deficient embryos the otic placode is larger, due to a combination of two factors: 1) an elongation of Wnt-reporter-positive, anterior regions of the placode and 2) an enlargement of a posterior-ventrolateral, Wnt-reporter-negative domain, which showed a greater magnitude increase in size compared to Wnt-reporter-positive regions.

\section{Spry genes function to limit Wnt signaling activity during early inner ear development}

Since both Wnt8a expression and TCF/Lef-lacZ reporter activity were elevated in Spry-deficient embryos, we hypothesized that Spry1 and Spry2 function by limiting Wnt signaling activity in approximately $80 \%$ of the otic placode where TCF/Lef-lacZ reporter is active. To functionally test this hypothesis, we genetically reduced Wnt signaling levels in Spry-deficient embryos by crossing-in a null allele of the gene that encodes for $\beta$-catenin, a Wnt signaling effector $\left(\right.$ Spry $^{-/-}$; Spry $2^{-/-} ; \beta$ - catenin $^{-/+}$ embryos, referred to below as "experimental" embryos). Littermate Spry-deficient embryos, with wild-type gene dosage of $\beta$-catenin $\left(\right.$ Spry $_{1}^{-/-}$; Spry $2^{-/-}$; $\beta$-catenin ${ }^{+/+}$embryos, referred to as "Spry-deficient controls") were used as reference controls of Spry-deficient otic phenotypes. Littermate Spry-heterozygous $\left(\operatorname{Spry1} 1^{-/+} ; \operatorname{Spry}^{-/+} ; \beta-\right.$ catenin $^{+/+}$) and Spry- $\beta$-catenin-triple heterozygous

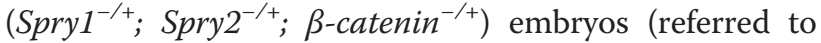
below as "heterozygous controls") were indistinguishable from wild-type embryos and from each other (data not shown) and were used as normal reference controls.

The earliest otic phenotype that we observed in Sprydeficient embryos was an enlargement of the otic placode [18]. To visualize the otic placode in experimental, Spry-deficient, and heterozygous control embryos, we examined expression of Pax8, an early marker of the otic placode [20], in $9-11 \mathrm{~s}$ embryos. In experimental embryos, the domain of $\operatorname{Pax} 8$ expression in the otic placode looked just as enlarged as in Spry-deficient controls (compare Fig. 5c with Fig. 5a and b; $n=4$ experimental embryos, $n=5$ Spry-deficient control embryos, $n=7$ heterozygous controls). Other genes whose expression patterns mark the otic placode were not suitable for our analysis: the expansion of the Pax2 expression domain in the otic placode in Spry-deficient embryos is incompletely penetrant [18]; expression of $H m \times 3$ is reduced in Spry-deficient embryos [42]; and $D l x 5$ expression is tightly regulated by Wnt signaling [43]. Thus, we examined the expression pattern of Foxi2, which is expressed in cranial epidermis, but is excluded from the otic placode [44]. At $12-14 \mathrm{~s}$, all Spry-deficient controls $(n=6)$ had enlarged Foxi2-negative domains, suggesting the presence of an enlarged otic placode (compare Fig. 5e to Fig. 5d; $n=18$ heterozygous controls). In $57 \%$ (4/7) of experimental embryos, the Foxi2-negative domains were more similar in size to heterozygous controls (compare Fig. $5 f$ to Fig. 5d). Whereas in $43 \%$ (3/7) of experimental embryos, the Foxi2-negative domains appeared more similar in size to Spry-deficient controls (data not shown). To directly examine whether the size of the otic placode was rescued in experimental embryos, we embedded and sectioned embryos to visualize the otic placode anatomically as a thickened epithelium that does not express Foxi2. We measured the anterior-posterior length of the otic placode - a metric that was increased in Spry-deficient embryos (see Fig. 4f) - by multiplying the number of sections containing an otic placode by the thickness of each section $(10 \mu \mathrm{m})$. Only the subset of experimental embryos in which the Foxi2-negative domain appeared similar to heterozygous controls by whole-mount in situ hybridization was analyzed. Average anterior-posterior lengths of the otic placode in experimental embryos were comparable to lengths in heterozygous controls (Fig. $5 \mathrm{j}, n=7$ experimental placodes, $n=6$ heterozygous placode, $p=0.12$ ), indicating restoration of otic placode expansions in this subset of experimental embryos. In both experimental and heterozygous embryos, average anterior-posterior otic placode lengths were significantly smaller than lengths in Spry-deficient controls (Fig. 5j, $n=8$ Spry-deficient placodes, $p<0.001$ for both comparisons). Thus, by assessment of the Foxi2-negative domain and direct measurement of the otic epithelium in histological sections, the otic placode/cup size is partially rescued in experimental embryos by $12-14$ s. The lack of rescue of the otic Pax8 expression domain in $9-11 \mathrm{~s}$ experimental embryos was not investigated further, but suggests the possibility that formation of the early otic placode is less sensitive to $\beta$-catenin gene dosage.

To examine morphogenesis of the otic epithelium, we stained whole mount embryos with E-cadherin antibody. Consistent with our previous observations [18], in heterozygous controls, by $19 \mathrm{~s}$ the rim of the otic cup appeared constricted and in the process of closure and by $24 \mathrm{~s}$ the otic cup was completely closed (Fig. 5g, $n=10$ ). In contrast, in Spry-deficient controls, at $19 \mathrm{~s}$, the otic cup was wide open, with no indication of closure. By $21-22 \mathrm{~s}$, otic cup closure initiated, but remained open past $26 \mathrm{~s}$ (Fig. 5h, $n=$ 
10). In $60 \%(3 / 5)$ of experimental embryos, E-cadherin staining revealed that closure of the otic cup had progressed further than any Spry-deficient control embryo examined, but was not as fully closed as in heterozygous controls (Fig. 5i). In the remaining $40 \%(2 / 5)$ of experimental embryos, the otic cup appeared more similar to Spry-deficient embryos.

Finally, we investigated whether incomplete rescue of Spry-deficient phenotypes was due to variability in reduction of Wnt signaling in embryos missing one functional allele of $\beta$-catenin. We produced embryos that were either wild-type or heterozygous for $\beta$-catenin, which also carried the TCF/Lef-lacZ reporter allele $\left(\beta\right.$-catenin ${ }^{++} ;$TCF/LeflacZ/+ and $\beta$-catenin ${ }^{-/+}$; TCF/Lef-lacZ/+ embryos) and measured Wnt reporter activity. We found that Wnt signaling was reduced in $\beta$-catenin ${ }^{-/+}$; TCF/Lef-lacZ/+ embryos, but to a variable extent. Whereas in $\beta$-catenin ${ }^{+/}$; TCF/ Lef-lacZ/+ control embryos, reporter activity was high in the majority of embryos (see Additional file 2: Figure S2A, D), in $\beta$-catenin ${ }^{-/+} ;$TCF/Lef-lacZ/+ embryos, reporter activity was variable, ranging from low to high (Additional file 2: Figure S2B - D). This variability in Wnt signaling activity in embryos missing one functional allele of $\beta$-catenin could contribute to the incompletely penetrant rescue of Spry-deficient phenotypes.

Combined, these data suggest that reduction of $\beta$-catenin gene dosage in $\operatorname{Spry}^{--}{ }^{-}$; Spry $2^{-/-}$mutant embryos can partially rescue the otic placode/cup defects observed. These data provide strong genetic evidence that Spry regulation of Wnt signaling levels during early inner ear development is functionally significant.

\section{Discussion}

\section{Timing and nodes of cross talk between FGF and Wnt signaling during otic fate specification}

Timing and pattern of Spry gene expression and Wnt reporter activity are consistent with the interpretation that FGF signaling precedes and is active in more pre-otic cells. Substantial evidence indicates that FGF signaling is required at multiple steps during the determination of otic fate, beginning with the specification of the PPR, from which all cranial placodes are derived $[2,4,6,8]$. In multiple organisms, expression of Sprouty genes are induced by FGF signaling, and serves as a read-out of FGF signaling activity [45-48]. This appears to be the case during specification of otic placode fate: Spry1 gene expression is absent in the dorsal OEPD in $\mathrm{Fgf3}^{-/-}$; Fgf $10^{-/-}$double mutants [29], and Spry1 and Spry2 expression in the chick is induced by the addition of FGF to early ectodermal cultures [49]. Our data that Spry1 and Spry2 are expressed continuously throughout PPR, OEPD and otic placode stages is consistent with the interpretation that FGF signaling functions during each of these stages. Furthermore, FGF signaling levels must be precisely modulated by
SPRYs or other negative regulators of RTK signaling, such as the dual-specificity phosphates $[50,51]$, for proper otic specification $[15,18,25]$.

Based upon the activity of the TCF/Lef-lacZ Wnt signaling reporter, it is thought that Wnt signaling is required after specification of the OEPD in the posterior PPR, to influence otic vs. epidermal cell fate decisions [14]. We demonstrate that Wnt reporter activity was detected in a subset of the Spry-expression domain, beginning at the OEPD stage. When cellular response to FGF signaling was upregulated in Spry-deficient embryos, both the size of the Wnt reporter expression domain and intensity of Wnt reporter staining were increased in the OEPD (see Fig. $4 \mathrm{a}-\mathrm{b}$ ). We found that Wnt8a expression was expanded in the hindbrain adjacent to the OEPD in Spry-deficient embryos [18], as well as in embryos in which Spry1 and Spry2 have been combinatorially inactivated (see Fig. 2). However none of the other genes that encode Wnt ligands or receptors that are known to be expressed at the OEPD stage - Wnt1, Wnt3, Wnt3a, Wnt5a, Wnt5b, Wnt6, Wnt7b, Fzd1, $F z d 3, F z d 7$, and $F z d 8$ - were up-regulated in Spry-deficient embryos. The expansion and increase in Wnt reporter activity in Spry-deficient embryos could be due to the expansion of Wnt8a expression alone. Alternatively, the increase in Wnt reporter activity could also be due to changes at other points in the Wnt signaling pathway.

Cross talk between the Wnt and FGF signaling pathways can occur downstream of receptor activation at common target proteins, or nodes (see [52] for review). One such node is GSK3 $\beta$, which phosphorylates cytoplasmic $\beta$ catenin to target it for proteasome-mediated degradation [53]. Wnt signaling inactivates GSK3 $\beta$ via phosphorylation, allowing $\beta$-catenin to enter the nucleus, bind members of the TCF/Lef family of transcription factors, and activate transcription of target genes (see [54-57] for recent reviews). FGF signaling can also inactivate GSK3 $\beta$ via phosphorylation by AKT $[53,58]$. Thus, by activation of the PI3K-AKT cascade, leading to inactivation of GSK3 $\beta$, FGF signaling can induce nuclear translocation of $\beta$ catenin. Inhibition of Sprouty gene function can lead to increased levels of phosphorylated AKT in certain cellular contexts [59], suggesting that in the inner ear, it may be possible for synergy between the Wnt and FGF signaling pathways to occur through phosphorylation of GSK3 $\beta$. In addition to its transcriptional function, $\beta$-catenin is a component of the adherens junction, where it links cadherins to the actin cytoskeleton [60]. In the mouse primitive streak, Fgfr1 indirectly promotes Wnt signaling by downregulating E-cadherin expression through expression of the Snail transcriptional repressor [61]. Reduced levels of E-cadherin allow for increased amounts of $\beta$-catenin to translocate to the nucleus and activate Wnt target genes. However, by immunohistochemistry, we did not detect a 
dramatic reduction of E-cadherin staining in the otic placode/cup in Spry-deficient embryos (see Fig. 5h and data not shown). Thus, it is unlikely that increased FGF signaling in Spry-deficient embryos relieves E-cadherinmediated sequestration of $\beta$-catenin in the cytoplasm, thus promoting Wnt signaling. Finally, cross talk between the FGF and Wnt signaling pathways can occur at the level of transcription. In Drosophila, the ETS-domain transcriptional repressor Anterior open (Aop, or Yan) competes with the ETS-domain transcriptional activator Pointed (Pnt) to repress gene expression downstream of RTKs [62]. Phosphorylation by MAPK leads to activation of Pnt and inactivation of Aop, resulting in target gene expression [63]. In addition, Aop represses gene expression downstream of Wingless (Wg, the Wnt-1 ortholog), through interaction with Armadillo (Arm, the $\beta$-catenin ortholog) [64], leading to transcriptional repression of Wg pathway components [65]. In the Drosophila tracheal system, inactivation of Aop by MAPK leads to de-repression of both FGF and Wntregulated genes, thus integrating both FGF and Wnt signaling at the level of a single transcriptional repressor [66]. Thus, it is possible that a variety of cellular targets downstream of FGF signaling can also cross talk with the Wnt signaling pathway to ultimately increase TCF/Lef-lacZ reporter activity in Spry-deficient embryos.

\section{FGF and Wnt signaling cooperate during early inner ear development}

When FGF signaling levels are elevated in a Spry gene dosage series, expansion of the otic placode and the $W n t 8 a$ expression domain correlate. The functional significance of this correlation is unclear, since in the mouse, Wnt8a is not required for otic placode formation, although it may play a role in combination with multiple Wnt genes [30]. However, in multiple organisms ectopic expression of Wnt can induce the formation of ectopic or enlarged otic vesicles [16, 21, 23, 27]. In some cases, ectopic induction of otic tissue by Wnt overexpression may be indirect: early over-expression of Wnt leads to posteriorization of the embryo, leading to ectopic expression of both Fgf genes and otic competence factors $[21,23]$. Later activation of Wnt signaling, during specification of the otic placode, leads to expansion of the otic domain at the expense of neighboring epidermal or epibranchial cells $[14,16]$. Thus, it is possible that in Spry-deficient embryos, elevated Wnt8a expression, in combination with elevated response of tissue to FGF signaling due to loss of SPRY negative regulation, is sufficient to ectopically direct more cells to an otic fate. Furthermore, the correlation between expansion of the otic placode and expansion of the Wnt8a hindbrain expression domain in a Spry gene dosage series is consistent with the possibility that FGF and Wnt signaling cooperate during otic placode formation.

We demonstrate that cross talk between the FGF/SPRY and Wnt signaling pathways is functionally required by performing a dominant genetic interaction experiment. Genetic reduction of $\beta$-catenin gene dosage in Spry-deficient embryos (Spry1 $1^{-/-}$; Spry $2^{-/-} ; \beta$-catenin ${ }^{-/+}$embryos) resulted in partial restoration of the normal Foxi2 expression pattern and otic placode size. This indicates that otic (Foxi2-negative) vs. epidermal (Foxi2-positive) cell fate decisions occur more normally in Spry-deficient embryos in which $\beta$-catenin levels are reduced than in Spry-deficient embryos alone. Furthermore, closure of the otic cup was partially rescued in Spry-deficient embryos in which $\beta$-catenin levels were genetically reduced. The delay in otic cup closure observed in Spry-deficient embryos could be due to the increased size of the otic placode and/or defects in morphogenesis of the otic cup during invagination [18, 42]. Similarly, increased size of the otic placode and defects in otic cup closure are observed in embryos in which an activated mutant form of $\beta$-catenin is expressed using the Pax2-Cre driver [14]. Thus, partial genetic rescue of the otic cup closure defect in Spry-deficient embryos missing one copy of the $\beta$-catenin gene suggest that Spry genes and $\beta$ catenin function in the same cellular processes that lead to proper closure of the otic cup. Combined, the genetic rescue observed suggests that $\beta$-catenin and Spry genes function in the same genetic pathway during early inner ear development, and demonstrate that aspects of FGF/SPRY signaling in the otic placode are mediated, directly or indirectly, by $\beta$-catenin.

\section{FGF-independent characteristics of the Wnt signaling response}

Although Spry genes and $\beta$-catenin function in the same genetic pathway in early inner ear development, multiple characteristics of Wnt reporter activity are independent of Spry gene function. First, in Spry-deficient embryos, the timing of onset of Wnt reporter activity was unaffected. Thus, elevated response of tissue to FGF does not generate a precocious response to Wnt signals. The timing of onset of Wnt signaling may be controlled independently of FGF signaling. This possibility is consistent with the finding that expression of the genes that encode candidate Wnt ligands and receptors are unaffected in Spry-deficient embryos (Wnt1, Wnt3, Wnt3a, Wnt5a, Wnt5b, Wnt6, Wnt7b, Fzd1, Fzd3, Fzd7, and Fzd8) or Fgf-deficient embryos (Wnt1, Wnt3a, Wnt6 [29]). Alternatively, onset of Wnt signaling may be controlled upstream of FGFR activation, by the spatial distribution and activity of FGF ligands. The finding that Wnt8a expression is reduced or absent in embryos in which Fgf3 and Fgf1O have been inactivated is consistent with this possibility [29]. 
Another characteristic of the Wnt signaling response that is independent of Spry gene function is the spatial distribution of the Wnt responsive domain. In the OEPD and otic placode, Wnt reporter activity was detected in anterior and dorsal regions, but was absent from a posterior-ventrolateral region. Although the Wnt reporter domain was larger in Spry-deficient embryos, it was still localized to anterior and dorsal regions. Thus, the spatial localization of Wnt ligands and receptors must be unaffected by increased tissue-response to FGF. Interestingly, although the size of the Wnt reporter domain increased in Spry-deficient embryos, the size of the reporter-negative domain increased even more (1.72-fold for the reporternegative domain vs. 1.36-fold for the reporter-positive domain). Therefore, in Spry-deficient embryos, the entire otic placode is larger due to increased response of pre-otic ectoderm to FGF. However, expansion of the Wntreporter-positive domain is more constrained than the Wnt-reporter-negative region. One possible explanation for the size constraint of the Wnt-reporter-positive domain is that multiple signals, including FGF and Wnt, are required for otic fate specification in this domain and the effective amount of these signals is limiting. Stringent cross talk between the FGF and Wnt signaling pathways may be one mechanism that ensures that otic fate specification in the anterior and dorsal domains is tightly regulated. For the posterior-ventrolateral domain, the effective amount of signals, including FGF, that regulate otic specification must be less stringently controlled. Alternatively, intrinsic anterior-posterior differences may affect the ability of the pre-otic ectoderm to respond to FGF and Wnt inductive signals. These differences may be influenced by other signaling pathways such as retinoic acid [67] or BMP [68].

The role of FGF signaling in the otic placode may be analogous to its role in the developing vertebrate limb bud. During limb development, FGFs, expressed in the apical ectodermal ridge (AER), have dual functions: 1) to specify the initial progenitor pool for all of the skeletal elements of the limb and 2) in conjunction with other signaling pathways, to expand these progenitor pools, with distal elements more sensitive to loss of AER-FGF function than proximal skeletal elements $[69,70]$. Similarly, in the otic placode, the FGF/SPRY pathway is required for specification of all otic progenitors. However, response of different regions of the otic placode to increased FGF signaling differs, and may depend on differential requirements for other signaling pathways, including Wnt. Recently, genomic approaches have been completed to identify FGF-regulated genes in the otic placode [29, 49]. To fully understand heterogeneity of the otic progenitor domain, the challenge will be to comprehensively define the distinct phenotypic responses and transcriptional targets that are activated and repressed by FGF alone, Wnt alone, and combinations of these and other signaling molecules during specification and patterning of the otic placode.

\section{Conclusions}

In this study, we investigated cross talk between FGF and Wnt signaling during specification and early morphogenesis of the otic placode. We show that FGF signaling precedes and is active in more cells of the OEPD and otic placode than Wnt signaling. Furthermore, we provide in vivo evidence that FGF signaling activates the Wnt signaling pathway upstream of TCF/Lef transcriptional activation. FGF regulation of the Wnt signaling pathway is functionally relevant, since early inner ear defects in Sprydeficient embryos can be genetically rescued by reducing the gene dosage of $\beta$-catenin. Thus, as in chick, Xenopus, and zebrafish, FGF and Wnt signals cooperate during otic specification in the mouse. However, we found that in Spry-deficient embryos, the Wnt-reporter-positive domain increases in size, but not to the same extent that the Wntreporter-negative domain expands. This result suggests that although otic specification is globally regulated by FGF signaling, otic specification of cells in which both FGF and Wnt signaling are active is more tightly regulated.

\section{Additional files}

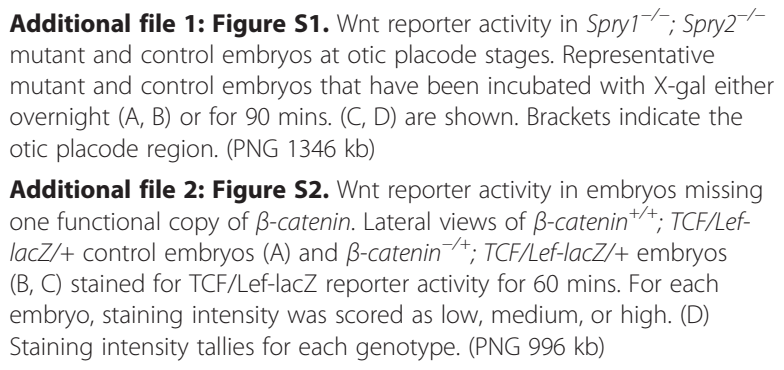

Additional file 2: Figure S2. Wnt reporter activity in embryos missing one functional copy of $\beta$-catenin. Lateral views of $\beta$-catenin ${ }^{+/+}$; TCF/LeflacZ/+ control embryos (A) and $\beta$-catenin ${ }^{-/+}$; TCF/Lef-lacZ/+ embryos $(B, C)$ stained for TCF/Lef-lacZ reporter activity for 60 mins. For each embryo, staining intensity was scored as low, medium, or high. (D) Staining intensity tallies for each genotype. (PNG 996 kb)

\section{Abbreviations}

FGF: Fibroblast growth factor; GSK3 $\beta$ : glycogen synthase kinase $3 \beta$; MO: Morpholino; OEPD: Otic-epibranchial progenitor domain; PPR: Pan-placodal region; RTK: Receptor tyrosine kinase; Spry: Sprouty.

\section{Competing interests}

The authors declare that they have no competing interests.

\section{Authors' contributions}

KDW and KS conceived of and designed the experiments. KDW, AAMR, JZ and KS performed the experiments and analyzed the data. KDW and KS wrote the manuscript. All authors read and approved the final manuscript.

Authors' information

Not applicable.

\section{Acknowledgements}

We wish to thank Dr. Daniel Dufort for generously providing the TCF/Lef-lacZ transgenic mice. Drs. Suzi Mansour, Lisa Urness, Takahiro Ohyama, Andy Groves, and Gail Martin for providing in situ probes; and Dr. Ramani Ramchandran for unrestricted use of the plastic microtome in his laboratory. This work was supported by N.I.H. grant DC010387 (to K. S.). 
Received: 5 February 2015 Accepted: 18 September 2015

\section{Published online: 06 October 2015}

\section{References}

1. Parr BA, Shea MJ, Vassileva G, McMahon AP. Mouse Wnt genes exhibit discrete domains of expression in the early embryonic CNS and limb buds. Development. 1993;119:247-61.

2. Chen J, Streit A. Induction of the inner ear: Stepwise specification of otic fate from multipotent progenitors. Hear Res. 2013;297(C):3-12.

3. Yamaguchi TP, Bradley A, McMahon AP, Jones S. A Wnt5a pathway underlies outgrowth of multiple structures in the vertebrate embryo. Development. 1999;126:1211-23.

4. Groves AK, Fekete DM. Shaping sound in space: the regulation of inner ear patterning. Development. 2012;139:245-57.

5. Takada S, Stark KL, Shea MJ, Vassileva G, McMahon JA, McMahon AP. Wnt-3a regulates somite and tailbud formation in the mouse embryo. Genes Dev. 1994:8:174-89.

6. Ladher RK, O'Neill P, Begbie J. From shared lineage to distinct functions: the development of the inner ear and epibranchial placodes. Development. 2010;137:1777-85.

7. Freyer $L$, Aggarwal $V$, Morrow BE. Dual embryonic origin of the mammalian otic vesicle forming the inner ear. Development. 2011;138:5403-14.

8. Ohyama T, Groves AK, Martin K. The first steps towards hearing: mechanisms of otic placode induction. Int J Dev Biol. 2007;51:463-72.

9. Groves AK, Labonne C. Setting appropriate boundaries_Fate, patterning and competence at the neural plate border. Dev Biol. 2014;389:2-12.

10. Schlosser G. Early embryonic specification of vertebrate cranial placodes. WIREs Dev Biol. 2014:3:349-63.

11. Borello U, Buffa V, Sonnino C, Melchionna R, Vivarelli E, Cossu G. Differential expression of the Wht putative receptors Frizzled during mouse somitogenesis. Mech Dev. 1999;89:173-7.

12. Schneider-Maunoury S, Pujades C. Hindbrain signals in otic regionalization: walk on the wild side. Int J Dev Biol. 2007:51:495-506.

13. Schimmang T. Expression and functions of FGF ligands during early otic development. Int J Dev Biol. 2007;51:473-81.

14. Ohyama T. Wnt signals mediate a fate decision between otic placode and epidermis. Development. 2006;133:865-75.

15. Freter S, Muta Y, Mak S-S, Rinkwitz S, Ladher RK. Progressive restriction of otic fate: the role of FGF and Wnt in resolving inner ear potential. Development. 2008;135:3415-24.

16. Mccarroll MN, Lewis ZR, Culbertson MD, Martin BL, Kimelman D, Nechiporuk AV. Graded levels of Pax2a and Pax8 regulate cell differentiation during sensory placode formation. Development. 2012;139:2740-50.

17. Mohamed OA, Clarke HJ, Dufort D. Beta-catenin signaling marks the prospective site of primitive streak formation in the mouse embryo. Dev Dyn. 2004;231:416-24.

18. Mahoney Rogers AA, Zhang J, Shim K. Sprouty1 and Sprouty2 limit both the size of the otic placode and hindbrain Wnt8a by antagonizing FGF signaling. Dev Biol. 2011;353:94-104.

19. Jayasena CS, Ohyama T, Segil N, Groves AK. Notch signaling augments the canonical Wnt pathway to specify the size of the otic placode. Development. 2008;135:2251-61

20. Pfeffer PL, Gerster T, Lun K, Brand M, Busslinger M. Characterization of three novel members of the zebrafish Pax2/5/8 family: dependency of Pax5 and Pax8 expression on the Pax2.1 (noi) function. Development. 1998;125:3063-74.

21. Phillips BT. A direct role for Fgf but not Wnt in otic placode induction. Development. 2004:131:923-31.

22. Hans $S$, Westerfield $M$. Changes in retinoic acid signaling alter otic patterning. Development. 2007;134:2449-58.

23. Bajoghli B, Aghaallaei N, Jung G, Czerny T. Induction of otic structures by canonical Wnt signalling in medaka. Dev Genes Evol. 2009;219:391-8.

24. Gutknecht D, Fritzsch B. Lithium can transform ear placodes of Xenopus into multiple otic vesicles connected by tubes. Naturwissenschaften. 1990;77:235-7.

25. Padanad MS, Bhat N, Guo B, Riley BB. Conditions that influence the response to Fgf during otic placode induction. Dev Biol. 2012:364:1-10.

26. Domínguez-Frutos E, Vendrell V, Alvarez Y, Zelarayan LC, López-Hernández I, Ros $M$, et al. Tissue-specific requirements for FGF8 during early inner ear development. Mech Dev. 2009;126:873-81.

27. Ladher RK. Identification of synergistic signals initiating inner Ear development. Science. 2000;290:1965-7.
28. Park B-Y, Saint-Jeannet J-P. Hindbrain-derived Wnt and Fgf signals cooperate to specify the otic placode in Xenopus. Dev Biol. 2008;324:10821.

29. Urness LD, Paxton CN, Wang X, Schoenwolf GC, Mansour SL. FGF signaling regulates otic placode induction and refinement by controlling both ectodermal target genes and hindbrain Wnt8a. Dev Biol. 2010;340:595-604

30. Vendrell V, Vázquez-Echeverría C, López-Hernández I, Alonso BD, Martinez S,

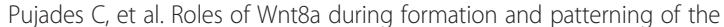
mouse inner ear. Mech Dev. 2013;130:160-8.

31. Basson M, Akbulut $S$, Watsonjohnson J, Simon R, Carroll T, Shakya R, et al. Sprouty1 Is a Critical Regulator of GDNF/RET-Mediated Kidney Induction. Dev Cell. 2005;8:229-39.

32. Shim K, Minowada G, Coling DE, Martin GR. Sprouty2, a mouse deafness gene, regulates cell fate decisions in the auditory sensory epithelium by antagonizing FGF signaling. Dev Cell. 2005;8:553-64.

33. Brault V, Moore R, Kutsch S, Ishibashi M, Rowitch DH, McMahon AP, et al. Inactivation of the beta-catenin gene by Wnt1-Cre-mediated deletion results in dramatic brain malformation and failure of craniofacial development. Development. 2001;128:1253-64.

34. Lewandoski M, Meyers EN, Martin GR. Analysis of Fgf8 gene function in vertebrate development. Cold Spring Harb Symp Quant Biol. 1997:62:159-68.

35. Marone M, Mozzetti S, De Ritis D, Pierelli L. Semiquantitative RT-PCR analysis to assess the expression levels of multiple transcripts from the same sample. Biol Procedures ... 2001;3:19-25.

36. Metzger RJ, Klein OD, Martin GR, Krasnow MA. The branching programme of mouse lung development. Nature. 2008;453:745-50.

37. Shim K. Vibratome sectioning for enhanced preservation of the cytoarchitecture of the mammalian organ of Corti. J Visual Exp. 2011;52:e2793

38. Wright TJ. Fgf3 and Fgf10 are required for mouse otic placode induction. Development. 2003;130:3379-90.

39. Alvarez Y. Requirements for FGF3 and FGF10 during inner ear formation. Development. 2003;130:6329-38.

40. Mansour SL, Goddard JM, Capecchi MR. Mice homozygous for a targeted disruption of the proto-oncogene int-2 have developmental defects in the tail and inner ear. Development. 1993;117:13-28.

41. Min H, Danilenko DM, Scully SA, Bolon B, Ring BD, Tarpley JE, et al. Fgf-10 is required for both limb and lung development and exhibits striking functional similarity to Drosophila branchless. Genes Dev. 1998;12:3156-61.

42. Zhang J, Wright KD, Mahoney Rogers AA, Barrett MM, Shim K. Compensatory regulation of the size of the inner ear in response to excess induction of otic progenitors by fibroblast growth factor signaling. Dev Dyn. 2014;243:1317-27.

43. Riccomagno M, Takada S, Epstein D. Wnt-dependent regulation of inner ear morphogenesis is balanced by the opposing and supporting roles of Shh. Genes Dev. 2005;19:1612-23.

44. Ohyama T, Groves AK. Expression of mouse Foxi class genes in early craniofacial development. Dev Dyn. 2004;231:640-6.

45. Guy GR, Jackson RA, Yusoff P, Chow SY. Sprouty proteins: modified modulators, matchmakers or missing links? J Endocrinol. 2009;203:191-202

46. Cabrita MA, Christofori G. Sprouty proteins, masterminds of receptor tyrosine kinase signaling. Angiogenesis. 2008;11:53-62.

47. Mason J, Morrison D, Albertbasson M, Licht J. Sprouty proteins: multifaceted negative-feedback regulators of receptor tyrosine kinase signaling. Trends Cell Biol. 2006;16:45-54.

48. Kim HJ, Bar-Sagi D. Modulation of signalling by Sprouty: a developing story. Nat Rev Mol Cell Biol. 2004;5:441-50.

49. Yang L, O'neill P, Martin K, Maass JC, Vassilev V, Ladher R, et al. Analysis of FGF-dependent and FGF-independent pathways in otic placode induction. PLoS One. 2013;8:e55011.

50. Li C, Scott DA, Hatch E, Tian X, Mansour SL. Dusp6 (Mkp3) is a negative feedback regulator of FGF-stimulated ERK signaling during mouse development. Development. 2007;134:167-76.

51. Urness LD, Li C, Wang X, Mansour SL. Expression of ERK signaling inhibitors Dusp6, Dusp7, and Dusp9 during mouse ear development. Dev Dyn. 2008;237:163-9.

52. Dailey L, Ambrosetti D, Mansukhani A, Basilico C. Mechanisms underlying differential responses to FGF signaling. Cytokine Growth Factor Rev. 2005;16:233-47. 
53. Beurel E, Grieco SF, Jope RS. Glycogen synthase kinase-3 (GSK3): Regulation, actions, and diseases. Pharmacol Ther. 2015;148:114-31.

54. Niehrs C. nrm3470. Nat Rev Mol Cell Biol. 2012;13:767-79.

55. Lien WH, Fuchs E. Wnt some lose some: transcriptional governance of stem cells by Wnt/ -catenin signaling. Genes Dev. 2014;28:1517-32.

56. Clevers H, Loh KM, Nusse R. Stem cell signaling. An integral program for tissue renewal and regeneration: Wnt signaling and stem cell control. Science. 2014;346:1248012.

57. Holland JD, Klaus A, Garratt AN, Birchmeier W. Wht signaling in stem and cancer stem cells. Curr Opin Cell Biol. 2013;25:254-64.

58. Jope RS, Johnson GWW. The glamour and gloom of glycogen synthase kinase-3. Trends Biochem Sci. 2004;29:95-102.

59. Basson M, Watsonjohnson J, Shakya R, Akbulut S, Hyink D, Costantini F, et al. Branching morphogenesis of the ureteric epithelium during kidney development is coordinated by the opposing functions of GDNF and Sprouty1. Dev Biol. 2006;299:466-77.

60. Kemler R. From cadherins to catenins: cytoplasmic protein interactions and regulation of cell adhesion. Trends Genet. 1993;9:317-21.

61. Ciruna B, Rossant J. FGF signaling regulates mesoderm cell fate specification and morphogenetic movement at the primitive streak. Dev Cell. 2001;:37-49.

62. Rebay I. Keeping the receptor tyrosine kinase signaling pathway in check: lessons from Drosophila. Dev Biol. 2002;251:1-17.

63. Brunner D, Dücker K, Oellers N, Hafen E, Scholz H, Klämbt C. The ETS domain protein pointed-P2 is a target of MAP kinase in the sevenless signal transduction pathway. Nature. 1994;370:386-9.

64. Olson ER, Pancratov R, Chatterjee SS, Changkakoty B, Pervaiz Z, DasGupta R. Yan, an ETS-domain transcription factor, negatively modulates the Wingless pathway in the Drosophila eye. EMBO Rep. 2011;12:1047-54.

65. Webber JL, Zhang J, Cote L, Vivekanand P, Ni X, Zhou J, et al. The relationship between long-range chromatin occupancy and polymerization of the drosophila ETS family transcriptional repressor Yan. Genetics. 2013:193:633-49.

66. Caviglia S, Luschnig S. The ETS domain transcriptional repressor Anterior open inhibits MAP kinase and Wingless signaling to couple tracheal cell fate with branch identity. Development. 2013;140:1240-9.

67. Bok J, Raft S, Kong K-A, Koo SK, Drager UC, Wu DK. Transient retinoic acid signaling confers anterior-posterior polarity to the inner ear. Proc Natl Acad Sci. 2011:108:161-6.

68. Abelló G, Khatri S, Radosevic M, Scotting PJ, Giráldez F, Alsina B. Independent regulation of Sox 3 and $\mathrm{Lm} \times 1 \mathrm{~b}$ by FGF and BMP signaling influences the neurogenic and non-neurogenic domains in the chick otic placode. Dev Biol. 2010;339:166-178.

69. Sun X, Mariani FV, Martin GR. Functions of FGF signalling from the apical ectodermal ridge in limb development. Nature. 2002;418:501-8.

70. Mariani FV, Ahn CP, Martin GR. Genetic evidence that FGFs have an instructive role in limb proximal-distal patterning. Nature. 2008;453:401.

\section{Submit your next manuscript to BioMed Central and take full advantage of:}

- Convenient online submission

- Thorough peer review

- No space constraints or color figure charges

- Immediate publication on acceptance

- Inclusion in PubMed, CAS, Scopus and Google Scholar

- Research which is freely available for redistribution 